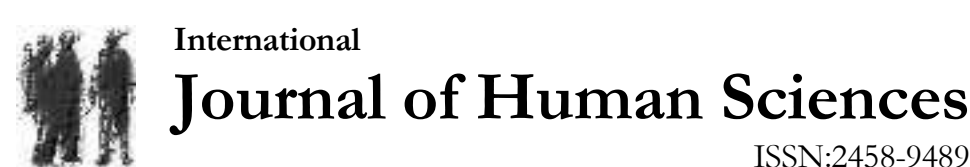

Volume 17 Issue 4 Year: 2020

\section{A systematic approach to athletic development}

\author{
Weston Gadient ${ }^{1}$ \\ Joe Deutsch ${ }^{2}$
}

\begin{abstract}
Since the dawn of social media, sports performance professionals have had the ability to share ideas and display training methodologies to anyone across the globe. Research problem/aim: The problem with this connectedness is much of this information is baseless. Coaches, athletes, and parents are too often misinformed, confused, and misled by fad exercise programs and gimmicks that can put their athletes under too much stress (physical and emotional) too fast, putting them at risk of injury. Findings: In order to be successful on the sporting field, athletes need to be able to make it to the playing field first. Sports performance specialists need to focus on long term development, not just pushing their athletes to the limit. Athletes need a structured training progression that builds a solid foundation of strength, endurance, and coordination to give them the tools to be successful in the weight room before placing them under a loaded bar or implementing advanced training techniques. With athletes at any level, high school, collegiate, or professional, even the most talented athletes on the field may not necessarily have a very strong background in the weight room. Conclusion: A systematic pattern of athletic development would allow adaptation in fundamental movement patterns and develops requisite physical qualities, and allow the athletes to advance safely and effectively.
\end{abstract}

Keywords: athletic development; athletes; sports performance; sports training; weight training.

\section{Introduction}

"Resistance training has been used as a means to augment muscular hypertrophy, muscular strength capacity, rate of force development production, and coordinated movement speed. Traditionally, each of these objectives was not thought to be mutually exclusive, and athletes were trained haphazardly to get bigger, stronger, and faster. However, as scientists and practitioners continue to collaborate, more empirical evidence suggests a need for specific training approaches to accommodate the various muscular fitness components. Training to stimulate adaptation within the muscular system exploits several synergistic physiological components that lend to increased force production, including neuromuscular, metabolic, and hormonal-capacity modifications" (Peterson, Alvar, \& Rhea, 2006, p. 867). For all athletes, the ability to summon these muscular fitness

\footnotetext{
${ }^{1}$ Graduate Assistant, North Dakota State University, Health, Nutrition, and Exercise Sciences, weston.gadient@,ndsu.edu

2 Professor of Physical Education and Coaching, North Dakota State University, joe.deutsch@,ndsu.edu

(iD) Orcid ID: 0000-0003-3094-6641
} 
components to perform a skill-related activity is essential for performing and competitive success. Just as inferior skill limits the extent of success in sports performance and coordination, for today's athlete the goal for a strength and conditioning program is to build strong, explosive, and injury resilient athletes but the approach to doing so can be a daunting task. Considerations for programming include injury history, the age group of the athletes, training experience, and the demands of their sport are just a few to name.

When programming for any group of athletes there should be a set structure of development that builds training qualities. This structure has been explained in many models including Strength and Conditioning coach Al Vermeil's Hierarchy of Athletic Development (left image), which places a hierarchical structure on the development of strength and conditioning qualities starting with Evaluation/Testing, Work Capacity (General Physical Preparedness or GPP), Strength, Explosive Strength, Elastic/Reactive Strength, and Speed. Durguerian, Piscione, Mathieu, and Lacome (2019), describe a Strength and Power Developmental Model in the article "Integrating Strength and Power Development in the Long-Term Athletic Development of Young Rugby Union Players" as comprised of four stages, each focused on educational, technical, and physical objectives, which should be achieved before progressing to the next level. These strategies allow the adjustment of the training process according to individual progress and maturation. Before engaging in intangible technical and physical developmental programs, it is important to remember that acquisition of fundamental movement skills and sufficient mobility are not only essential in building efficient sportspecific skills but also Resistance Training Skills Competency (RTSC).
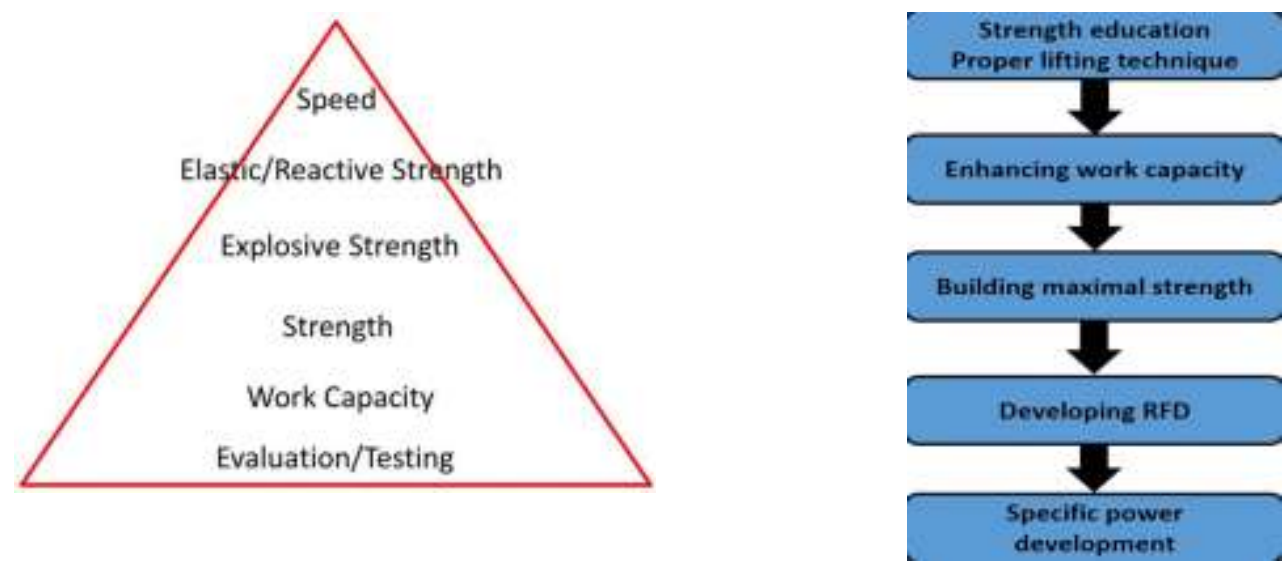

\section{The Hierarchy of Athletic Development}

The Model of the Hierarchy of Athletic Development describes an athlete's various physical qualities it occurs along a specific continuum and is initiated at the base of the pyramid; with the eventual progression to each ascending or succeeding physical quality levels once the criterion of the current physical quality level of training is achieved. More than one physical quality can be trained at a specific period of time. However, it is recommended that only one specific physical quality be the main emphasis at each corresponding stage of training. What transpires during the specific stages of each physical quality of development is the application of stress, which is essential to ensure the body adapts and the athlete continues concurrence upon this model of physical development. The appropriate level of programmed and applied stress will depend upon many factors such as the training experience of the athlete (i.e. novice vs advanced), the biological age of the athlete, the sex of the athlete, and medical history. What is particularity important is the second stage of the model, the stage of development of work capacity or the general preparatory period. An athlete must be prepared for the eventual demands and applied stress. Increased levels of programmed stress applied to an unprepared athlete will place the athlete at greater risk of injury. This approach to the development of athletes starts with athlete evaluation, a base of technical skill and enhancement of 
work capacity (GPP), the continued focus on development of strength is built to increase the rate of force development, to transfer to power and speed.

\section{Evaluation/Testing}

Prior to participating in any prescribed strength and conditioning program, athlete evaluation plays a critical role in assessing readiness. This evaluation takes into consideration prior training history or training experience, biological age, sex, and injuries or current health concerns. Just placing an athlete into a program and hoping they can handle it, is ill-advised and the cardinal rule of any practitioner "do no harm." Putting an athlete under excessive stress or assuming preparedness to execute any exercise movements places the athlete at risk of injury and places the coach at risk of being held responsible for the outcome. After receiving medical clearance, the coach is to assess the physical assets and deficits presented by the athlete, this will help determine the specific goals of the initial training program. When working with novice athletes it is especially important to teach the basic movements and perform them with sound technique. It is ultimately up to the coach to set the standard, teach the exercises, and refine technique. With intermediate and advanced athletes, assuming their competence in performing exercises, especially coming back from an extended break or a new phase of training is ill-advised. Even intermediate and advanced athletes need teaching, coaching, and technique refinement, it is an endless effort and is of utmost importance for keeping athletes safe in the weight room and maximizing performance.

\section{Developing Work Capacity or General Preparatory Period (GPP)}

After evaluation, work capacity is the foundational quality in the hierarchy and comprises an athlete's ability to work for a prolonged period maintaining quality and intensity of work and displaying appropriate recovery from the activity. This quality can be impacted by an athlete's previous exposure to formalized training or sport participation. It requires that athletes have a base level of ideal body composition and normal joint mechanics with no active injuries If any of these qualities are deficient, priority is given to bringing them up to at least an acceptable baseline level. Athletes must be able to demonstrate appropriate levels of strength endurance, core strength and stability, and aerobic capacity through bodyweight exercises or very light resistance. Prior to entering this system [next phases] (Parker, Miller, Panariello, \& Hall, 2018). The purpose behind the general preparatory period is to develop a foundational base for the athlete, develop starting strength and coordination, and fix any limiting factors that were seen in the evaluation.

\section{Develop Strength}

The GPP phase lays a foundation of endurance, strength, and mobility. Once the athlete is able to perform the prescribed program in the previous stage, the focus of training is then shifted toward strength development. "The purpose of this phase is to increase force production. Strength is the ability to exert maximal of the muscles that control particular body movements" (Bird, Tarpenning, \& Marino, 2005). "Strength is the base of all performance-based athletic development program. Strength is purely the ability to exert force. Power and speed are qualities that demand that strength be applied rapidly, as is the case in most organized sports. These qualities cannot be appropriately addressed without establishing a baseline of whole-body strength.” (Parker, et. al., 2018, p. 25). By making the athletes stronger, they will become more resilient to injury and have the tools necessary for the demands of their sport.

\section{Explosive Strength/Rate of Force Development}

Force is anything capable of changing an object's state of motion. Power is the rate at which energy is transferred, power is force multiplied by velocity. Improvement in power by a deficit of velocity or increasing the speed of movements without losing force allows the athlete to be able to use the strength and coordination gained in the previous stages and display it very quickly. The purpose of developing explosive strength/power is to produce a large amount of force (strength) and apply it 
quickly to overcome the demands of their sport. The most successful athletes are typically the most explosive. The competitors that can change direction quickly, hit/move objects, and/or opponents. This is the first step towards focused improvement on those abilities.

\section{Elastic/Reactive Strength}

Elastic/Reactive strength is the ability of tissues to absorb, store, and release energy. The more energy these tissues release, the faster and more powerful the movement. But instead of just looking at just the actions of muscles, consider the high level of elastic strength that can be produced by connective tissues, especially tendons. Tendons should not be thought of as simply rigid cables that connect muscle to bone. Tendons have elastic qualities that can assist the muscles in producing power by acting as "biological springs" that compress and elongate. The focus of this stage of development is to produce force while overcoming minimal load or none at all. The athletes are capable of applying force quickly while overcoming a substantial external load that quality was developed during training of explosive strength. Elastic/Reactive strength assists in the development of explosiveness, reaction, and elasticity. Moving light weights extremely fast or utilizing jumps and throws.

\section{Speed}

Speed is developed through the progression of all of the previously trained qualities. It is the outcome so to speak. Speed training is a skill that requires maximal effort and that max effort is very taxing to the nervous system. In the case of speed development, less is more. The purpose of this stage is to move the body quickly through the ranges of motion required of their sport.

\section{Strength and Power Developmental Model Training Principles Stage 1}

In this stage of development, the foundation is built focusing on global physical fitness. This period is crucial to address the educational and technical basis of resistance training for the proficient longterm development of strength and power. The athletes should have the opportunity to learn and practice the desired skills in a controlled environment. The acquisition of proper lifting techniques should be a prerequisite before attempts are made to enhance physical quality. Utilize full-body strength and power exercises. Volume and intensity are low to allow for correct teaching of lifting. During this time athletes and coaches should not look at how much they can lift, but rather at how well they can lift it. Training duration and volume are kept low to avoid excessive stimulation of the neuromuscular system and ensure the quality of training. Focusing on technical and postural skills does not mean that the other aspects of strength and power training will not be addressed. Basic resistance training exercises, such as military press and dumbbell pressing and pulling movements, and power exercises, such as bilateral low impact plyometrics or box jumps, should be integrated. This strategy will allow athletes to achieve technical mastery to optimize the development of hypertrophy and explosiveness during later stages. Postural and technical proficiency should remain a cornerstone of the strength training program, even following the first stage, and should always be the first aspect to consider when designing and adjusting training parameters (Durguerian, Piscione, Mathieu, \& Lacome, 2019).

\section{Stage 2}

This stage involves an increase in resistance training volume to enhance the athlete's working capacity. Duthie (18 states that work capacity is a prerequisite to any physical capacity development and should be driven by increasing training frequency, volume, and then intensity. Therefore, adding resistance training sessions in the training program is a primary step toward higher training loads. Resistance training volume is enhanced concomitantly through increased training frequency and the number of exercises and sets performed during each session. This training strategy is achieved by athletes performing 6-7 exercises in the session with 3-4 sets (warm-up sets not included) for each exercise. Nevertheless, increasing training volume should be progressive and individually adapted to optimize 
training adaptations (Lloyd \& Oliver, 2012). Training intensity is kept low to moderate and is adjusted according to technical and postural quality to promote high-quality movement. This principle is particularly important as new strengthening exercises (secondary and auxiliary movements) will be added to the training programs. The training session parameters will be adjusted to embrace these principles. Between 6 and 10 repetitions for each set should be the target zone because these not only allow the athlete to tolerate a higher training volume (high repetitions with increased training load compared with stage 1) but also to achieve a high quality of movement with progressively higher training loads $(60-70 \% 1 \mathrm{RM})$. However, prioritizing increased training volume is recommended, especially through a higher number of sets performed, before increasing training intensity.

\section{Stage 3}

Once work capacity had been developed, the training goal should focus on increasing maximal strength capacity. Strength development relies on structural and neural factors. When building longterm maximal strength, it has been proposed to put the initial emphasis on the development of structural strength-related adaptations and thereafter focus on neural adaptations. Stimulating muscle hypertrophy necessitates mechanical tension, metabolic stress, and adapted nutrient intakes (Crewther, Cronin, \& Keogh, 2006). The load and the time under tension, therefore, are of paramount importance. Resistance training programs that aim to optimize structural adaptations should activate the glycolytic metabolism (lactate and $\mathrm{H}+$ ions production) and induce muscular damage (Schoenfeld, 2010). This phenomenon is achieved through sets to failure to maximally activate muscle groups. Even if high- (60-80\% 1RM) and low-intensity (30-50\% 1RM) methods have been prescribed to promote muscular hypertrophy (Bird, Tarpenning, \& Marino, 2005), hightraining intensity would be more effective if achieving simultaneously both muscle growth and preparation for maximal strength training (Howe, Read, \& Waldron, 2017). A recent study has also shown that the global training volume was more important than the training method prescribed to stimulate increases in muscular size (Angleri, Ugrinowitsch, \& Libardi, 2017). Therefore, resistance training programs should be composed of high training volume (6-7 exercises, 3-5 sets, and 6-10 repetitions) with training intensity programmed between 60 and $80 \% 1 \mathrm{RM}$. The interset recovery duration also influences the metabolic stress induced by the training session. Short interset periods ( $<60$ seconds) increase metabolic stress, whereas longer interset periods (90-180 seconds) enable the use of higher training intensities and, thus, achieving stronger mechanical tension (Howe, et. al. 2017). Therefore, unless the athlete is in rehabilitation, moderate to high training intensity $(60-80 \% 1 \mathrm{RM})$, associated with long interset recovery (90-180 seconds), should be prioritized to address hypertrophy development and ensure adequate technical and physical preparation for maximal strength training. When focusing on neurological development, training repetitions should be kept between 1 and 5 repetitions per set, and the interset recovery period should be longer than for hypertrophy methods (180-300 seconds) to enable implementation of higher training intensity ( $>85 \% 1 \mathrm{RM}$ ) (Kraemer \& Ratamess, 2004). Accordingly, a recent study has proposed the use of the rating of perceived exertion, based on the repetitions in the reserve system, as a method to calibrate the training load of the prescribed exercises. This seems a pertinent tool for adjusting the parameters to the daily fitness level while maintaining the training goal (Helms, et. al. 2016). However, this training method requires training experience and should be introduced progressively to ensure that players have integrated this principle. When considering optimal strength development and fatigue management, the global training volume is an essential parameter and should be monitored weekly (Fyffe, Bishop, \& Stepto, 2014). Although maximal strength development is the main objective of this training phase, Olympicstyle weightlifting movements are still present in the training program. This strategy allows the athlete to master technical proficiency with progressively increasing loads and develop explosive strength. Similarly, low- to moderate-intensity level plyometrics, such as single- and double-leg hops, hurdle jumps, and jump squats should be incorporated in the training program to improve muscular power (Lloyd and Oliver, 2012) and prepare the athlete for the upcoming training stage.

\section{Stage 4}


Improvement in muscular power represents the final stage of the physical development pathway. Maximal power output depends on each movement pattern and individual strength levels but generally achieved with a load equivalent of $30-50 \%$ of maximal strength capacity (Baker, 1995). This phenomenon is highlighted by the force-velocity-power relationship

According to the principle of progressive overload, increasing training loads is necessary to improve strength and power capacity among high-level athletes (Fleck \& Kraemer, 2004). In other words, players need to train more to improve strength and power. Strength training frequency should comprise between 3 and 5 weekly sessions and be adjusted according to the competitive schedule. Different periodization strategies (e.g., linear periodization, block pattern, and undulating) should be considered to optimize training adaptations (Beaven, et. al. 2008). Training volume will be adjusted according to maintenance or developmental period. Through information gleaned from scientific recommendations (Schoenfeld, 2010) combined with practical experience, we advocate that a weekly training volume of 10-15 sets of maximal strength is needed to allow for upper- and lower-body strength maintenance. On the other hand, developing maximal strength and power capacities would require 15-30 sets per week and 20-30 sets per week, respectively. Besides traditional resistance training exercises, reducing the ROM, adding external resistance through the use of chains and elastic bands, also called variable resistance training, or alternating a maximal strength-oriented load with a lighter load (contrast method) have been shown to be very effective in stimulating neuromuscular adaptations and maximizing transfer in sport-specific performance (Rhea, Kenn, Peterson, Massey, Simão, Marin, Favero, Cardozo, \& Krein, 2016). Some authors also propose the use of movement velocity to provide visual feedback to the athlete and to adjust the load lifted according to movement velocity. This training method, called velocity-based training, has been shown to optimize maximal power output development (Jovanovic and Flanagan, 2014). When considering strength and power transfer, exercise specificity is an essential parameter (Young, 2006).

It is recommended that the implementation of power and explosive training sessions when athletes are physically and mentally fresh to ensure optimal stimulation of the neuromuscular system. During the competitive season, a minimal period of 48 hours after the game seems necessary for adequate neuromuscular recovery (West, Finn, Cunningham, Shearer, Jones, Harrington, Crewther, Cook, \& Kilduff, 2014). Power training exercises will typically include 3-5 sets with 1-5 repetitions for weightlifting movements and 3-8 repetitions for plyometric and ballistic movements (Janz, Dietz, \& Malone, 2008). Training intensity should be adjusted according to the part of the force-velocity curve toward which the exercise is focused (Newton and Kraemer, 1994).

\section{Conclusion}

The concept of developing talent and athleticism is the goal of many coaches and sport systems. Subsequently, strength and conditioning coaches can devote much of their time to adjusting training parameters and pedagogical approaches to tailor for individual differences and needs. A systematic approach to athletic development is crucial for every system of athletic development. As described in Al Vermeil's Hierarchy of Athletic Development the athletes are evaluated to assess readiness for exercise prescription, followed by work capacity to build general fitness, enhance coordination, and build a foundation of fitness to build upon. The next phase is strength, the focus is to enhance the rate of force development. The next stage is Power is the rate at which energy is transferred, power is force multiplied by velocity. Improvement in power by a deficit of velocity or increasing the speed of movements without losing force allows the athlete to be able to use the strength and coordination gained in the previous stages and display it very quickly. Once power is developed elastic strength becomes the focus this allows the connective tissue to absorb force and enhance elastic efficiency. The final stage is speed, speed is the outcome of all previous qualities and is then transferred to the field. Durguerian et. al (2019) describes building a specific developmental pathway that focuses on the training process and strategies to ensure continual long term development. The general pathway is divided into four main stages allowing for educational and technical efficiency in the first stage, enhancing work capacity in the second stage, building a high level of maximal strength in the third 
stage, and improving specific explosive strength and power capacity in the final stage. Besides these global objectives, the evolution of this training process (i.e., accessing from one stage to another) should be based on individual progression and not only on chronological and biological age. Finally, developing strength and power capacity represents a major challenge because of concomitant technical and energetic developmental needs. This type of training, recognized as concurrent training, has been shown to induce interferences in cellular signaling pathways, thereby limiting optimal adaptations in each quality. Beyond the sole development of maximal strength and power, the strength and conditioning coach will have to integrate these components into a more complex environment to maximize sport performance. In order to be successful on the sporting field, athletes need to be able to make it to the playing field first (Christianson \& Deutsch, 2012). Sports performance specialists need to focus on long-term development not just pushing their athletes to the limit. Developing a systematic pattern of athletic development would allow adaptation in fundamental movement patterns to occur and develops requisite physical qualities, and allow the athletes to advance safely and effectively throughout their athletic careers.

\section{References}

Angleri, V., Ugrinowitsch, C., \& Libardi, C. (2017). Crescent pyramid and drop-set systems do not promote greater strength gains, muscle hypertrophy, and changes on muscle architecture compared with traditional resistance training in well-trained men. European Journal of Applied Physiology, 117, 359-369.

Baker, D. (1995). Selecting the appropriate exercises and loads for speed and strength development. Strength and Conditioning Coach, 3, 8-16.

Beaven, M., Cook, C., \& Gill, N. (2008). Significant strength gains observed in rugby players after specific resistance exercise protocols based on individual salivary testosterone responses. Journal of Strength and Conditioning Research, 22, 419-425.

Bird, S., Tarpenning, K., \& Marino, F. (2005). Designing resistance training programme to enhance muscular fitness: A review of the acute programme variables. Sports Medicine, 35, 841-851.

Christianson, P. \& Deutsch, J. (2012). Making a case for early sports specialization. Journal of Youth Sports, 6(2), 3-6.

Crewther B., Cronin J., \& Keogh J. (2006). Possible stimuli for strength and power adaptation: Acute metabolic responses. Sports Medicine, 36, 65-78.

Durguerian, A., Piscione, J., Mathieu, B., \& Lacome, M. (2019). Integrating strength and power development in the long-term athletic development of young rugby union players. Strength and Conditioning Journal, 41(4), 18-33.

Fleck S. \& Kraemer W. (2004). Exercise prescription of resistance training. In: Designing Resistance Training Programs. 3rd ed. Champaign, IL: Human Kinetics, 149-260.

Helms, E., Cronin, J., Storey, A., \& Zourdos, M,. (2016). Application of the repetitions in reservebased rating of perceived exertion scale for resistance training. Strength and Conditioning Journal, $38,42-49$.

Howe, L., Read, P., \& Waldron, M. (2017). Muscle hypertrophy: A narrative review on training principles for increasing muscle mass. Strength and Conditioning Journal, 39, 72-81.

Janz, J., Dietz, C., \& Malone, M. (2008). Training explosiveness: weightlifting and beyond. Strength and Conditioning Journal, 30, 14-22.

Jovanovic, M. and Flanagan, E. (2014). Researched applications of velocity strength training. The Journal of Australian Strength and Conditioning, 22, 58-69.

Kraemer, W. and Ratamess, N. (2004). Fundamentals of resistance training: progression and exercise prescription. Medical Science and Sports Exercise, 36, 674-688.

Lloyd, R. and Oliver, L. (2012). The youth physical development model: A new approach to longterm athletic development. Strength and Conditioning Journal, 34, 61-72. 
Newton, R. and Kraemer, W. (1994). Developing explosive muscular power: implications for a mixed methods training strategy. Strength and Conditioning Journal 16, 20-31.

Parker, J., Miller, A., Panariello, R., \& Hall, J. (2018). The System: Soviet Periodization Adapted for the American Strength Coach. Aptos, CA: On Target Publication.

Peterson, M., Alvar, B., \& Rhea, M. (2006). The contribution of maximal force production to explosive movement among young collegiate athletes. The Journal of Strength and Conditioning Research, 20(4), 867.

Rhea, M., Kenn, J., Peterson, M., Massey, D., Simão, R., Marin, P., Favero, M., Cardozo, D., \& Krein, D. (2016). Joint-angle specific strength adaptations influence improvements in power in highly trained athletes. Human Movement Science, 17, 43-49.

Schoenfeld, B. (2010). The mechanisms of muscle hypertrophy and their application to resistance training. Journal of Strength and Conditioning Research, 24, 2857-2872.

West, D., Finn, C., Cunningham, D., Shearer, D., Jones, M., Harrington, B., Crewther, B., Cook, C., \& Kilduff, L. (2014). Neuromuscular function, hormonal, and mood responses to a professional rugby union match. Journal of Strength and Conditioning Research, 28, 194-200.

Young, W. (2006). Transfer of strength and power training to sports performance. International Journal of Sports Physiology and Performance 1, 74-83. 